\title{
Simulation of Locked-cycle Grinding of Multicomponent Feeds and its Implications for Stability and Control of Industrial Comminution Circuits
}

\author{
P.C. Kapur \\ Department of Metallurgial Engineering, Indian Institute of Technology Kanpur (India) \\ Rohit Verma \\ Department of Metallurgial Engineering, Indian Institute of Technology Kanpur (India)
}

B.V. Velamakanni

Dept of Materials Science and Mineral Engineering, University of California, Berkeley, CA 94720 (U.S.A )

D.W. Fuerstenau

Dept of Materials Science and Mineral Engineering, University of California, Berkeley, CA 94720 (U.S.A )

A mathematical algorithm is described for cycle-wise simulation of locked-cycle grinding tests in a ball mill using multicomponent feeds. The simulation is in good agreement with extensive experimental data for quartzite and limestone mixture feeds ranging in composition from 1:3 to 3:1. The simulation model is employed for generating transients in the locked-cycle tests by imposing either relatively large step changes or random fluctuations in a narrow band width on the composition of the feed, its fineness and the grinding time. Because locked-cycle tests mimic closed-loop grinding circuits with plug flow transport through the mill, the simulation provides useful insight into the stability and control of industrial comminution circuits where ore composition and hardness and the feed rate are invariably subjected to minor fluctuations on a more or less continuous basis, and occasionally to rather abrupt large changes.

\section{Introduction}

Locked-cycle grinding tests are widely used for assessing the grindability of industrial minerals and ores, for the design and scale-up of industrial mills, and for studying the likely behavior of industrial grinding circuits under different operating conditions [1-4]. These tests experimentally simulate on the laboratory scale a tumbling mill operating under plug flow conditions in a closed loop with a classifier which may or may not be perfect. It is well known that locked-cycle tests are time-consuming, laborious, and prone to experimental errors. 
Therefore, based on appropriate mathematical algorithms, a number of quite accurate simulation schemes have been proposed in order to economize on the experimental component of these tests [5-8],

In place of a single component feed, Venkataraman and Fuerstenau $[9,10]$ employed a calcite-quartz mixture feed for conducting fixed-time locked-cycle grinding tests. It is evident that multicomponent feeds comprising hard and soft minerals in different proportions should provide a more realistic model representation of the dynamics of a closed-circuit mill grinding heterogeneous industrial ores. These complex ores, of course, are of primary importance, both in terms of the tonnage ground and the comminution energy expended. On the other hand, compared to single-component feeds, much more experimental effort is required for lockedcycle grinding of mixture feeds. Not only is the number of cycles needed to attain steady-state significantly higher, but also it is necessary to analyze the composition of the recycling mass (or of the finished product) at the end of each cycle. Recently, Kapur and Fuerstenau [11] presented a number of mathematical algorithms for cycle-wise simulation of locked-cycle tests using mixture feeds, which on the whole performed quite satisfactorily when tested against the aforementioned experimental data of Fuerstenau and Venkataraman for 1:1 calcite-quartz feed. Even so, these algorithms were based on highly simplified versions of the more realistic models of grinding kinetics, had no provision for 'on-line' change of grinding time in the course of a simulation run and could not account for interaction phenomena between hard and soft particles except in a rather arbitrary sense.

Consequently, the aim of the present work is to derive a more general and versatile simulation algorithm for multicomponent feeds which incorporates an energy split factor for mixture grinding along the lines proposed recently by Kapur and Fuerstenau [12] and which can be employed for variable grinding times also. The algorithm has been validated against rather extensive locked-cycle grinding data for quartzite-limestone mixture feeds in 1:3, 1:1 and 3:1 weight ratios, including the transient behavior when a step change is imposed on the feed composition. Finally, this scheme has been employed to simulate the effects of random disturbances and unintentional changes in ore composition, size consist and feed rate on the recycle ratio in particular and on the stability of the locked-cycle system in general which, in turn, provides an interesting insight into the stability of a continuous closed-loop grinding circuit when subjected to similar disturbances.

\section{Simulation Algorithm}

As before [11], the mill transfer function used in the present simulation scheme is based on the G-H solution to the population balance grinding equation:

$$
R=r F \phi(t)
$$

And

$$
\phi(t)=\exp \left(G t+\frac{H t^{2}}{2}\right.
$$


where $R$ is weight of solids retained on the mesh-of-grind screen after a grinding time interval $t$, and $F$ is weight of mill feed having fraction $r$ which is coarser than the mesh-of-grind. The lumped grinding parameters $G$ and $H$ comprise the breakage rate and breakage distribution functions in the population balance model of grinding kinetics and the feed size distribution [13].

\section{Single-component Feed Case}

The simulation algorithm for locked-cycle tests using a single-component feed has already been derived by Kapur and Rahman [7] and is summarized here for the purpose of introducing the notation.

If $M$ is the total weight of mill feed, which remains fixed for every cycle, the new makeup feed for $n+1$ th locked-cycle is:

$$
F(n+1)=M-R(n)
$$

where $R(n)$, the amount retained on the mesh-of-grind and recycled after the nth cycle, is:

$$
\begin{aligned}
& R(n)=r \sum_{j=1}^{n} F(j) \phi\left(t_{j}\right) ; n=1,2, \ldots \\
& R(n)=0 ; n=0
\end{aligned}
$$

And

$$
t_{j}=(n-j+10) t
$$

Hence

$$
R(n)=r \sum_{j=1}^{n} F(j) \exp \left[G(n-j+1) t+\frac{H}{2}[n(n-j+1) t]^{2}\right]
$$

The percent circulating load ratio is by definition:

$$
\theta(n)=\frac{R(n-1-100}{f(n)} 100
$$

The simulation procedure entails recursive computations of the set of system variables $F(n), R(n)$, and $\theta(n)$ in these equations until the set converges to constant values at steadystate. A major advantage of this scheme is that for its implementation only two parameters, $G$ and $H$, are required, which can be estimated from a limited number of either batch grinding or locked-cycle tests.

\section{Multicomponent Feed Case}

The major factor in the case of multicomponent feed involves the interaction between soft and hard particles in the mill charge, as a result of which, depending on the charge composition, the grinding rates of mineral constituents are either somewhat accelerated or retarded $[9-11,14]$, This means that the mill transfer function, i.e. $G$ and $H$ in our case, varies 
continuously from cycle to cycle as the relative proportion of the minerals changes after each grinding cycle. If $m_{L}$ and $m_{Q}$ are weight fractions of component 1 (say limestone) and of component 2 (say quartzite) in the mixture feed, then at the start of the first cycle the mill feed comprises $F_{L}(1)$ and $F_{Q}(1)$ amounts of limestone and quartzite where:

$$
F_{L}(1)=m_{L} M
$$

And

$$
F_{Q}(1)=m_{Q} M
$$

If $r_{L}$ and $r_{Q}$ are the fractions of the two mineral feeds which are coarser than the meshof-grind, then the ground product contains the following amounts of limestone and quartzite in the recycle after the first cycle of grinding:

$$
R_{L}(1)=r_{L} F_{L}(1) \phi_{L}\left[t S_{L}(1)\right]
$$

And

$$
R_{Q}(1)=r_{Q} F_{Q}(1) \phi_{Q}\left[t S_{Q}(1)\right]
$$

where $S(1)$ is the energy split factor [12] for the first grinding cycle which in effect rescales the grinding time to some extent, depending on the composition of the mill charge in the first grinding cycle. The total recycle is:

$$
R(1)=R_{L}(1)+R_{Q}(1)
$$

And the make-up feed for the second cycle is:

$$
F(2)=M-R(1)
$$

Its composition is:

$$
F_{L}(2)=m_{L} F(2)
$$

And

$$
F_{Q}(2)=m_{Q} F(2)
$$

Therefore at the end of the second cycle, we have

$$
R_{L}(2)=r_{L} F_{L}(1) \phi_{L}\left[t S_{L}(1)+t S_{L}(2)\right]+r_{L} F_{L}(2) \phi_{L}\left[t S_{L}(2)\right]
$$

And

$$
R_{Q}(2)=r_{Q} F_{Q}(1) \phi_{Q}\left[t S_{Q}(1)+t S_{Q}(2)\right]+r_{Q} F_{Q}(2) \phi_{Q}\left[t S_{Q}(2)\right]
$$

Here again the energy split factor 5(2) for the second cycle depends on the charge composition, which in weight fractions is as follows: 
$M_{L}(2)=\frac{R_{L}(1)+F_{L}(2)}{M}$

And

$M_{Q}(2)=\frac{R_{Q}(1)+F_{Q}(2)}{M}$

It will be seen that the general algorithm for cycle-wise simulation of locked-cycle grinding tests becomes:

$$
\begin{aligned}
& R\left(n-1_{=R_{L}(n-1)}+R_{Q}(n-1)\right. \\
& F(n)=M-R(n-1) \\
& F_{L}(n)=m_{L} F(n) \\
& F_{Q}(n)=m_{Q} F(n) \\
& M_{L}(n)=\frac{R_{L}(n-1)+F_{L}(n)}{M} \\
& M_{Q}(n)=\frac{R_{Q}(n-1)+F_{Q}(n)}{M}=1-M_{L}(n) \\
& R_{L}(n)=r_{L} \sum_{j=1}^{n} F_{L}(j) \phi_{L}\left[t \sum_{k=j}^{n} S_{L}(k)\right] \\
& R_{Q}(n)=r_{Q} \sum_{j=1}^{n} F_{Q}(j) \phi_{Q}\left[t \sum_{k=j}^{n} S_{Q}(k)\right]
\end{aligned}
$$

where $S(k) s$ are some fucntions, $f$, of the composition of the $k$ th cycle, that is:

$$
S_{L}(k)=f_{L}\left[m_{L}(k)\right]
$$

And

$$
S_{Q}(k)=f_{Q}\left[m_{Q}(k)\right]
$$

The percent overall circulating load, which is of primary interest in the simulation of grinding circuits is:

$$
\theta(n)=\frac{R(n-1)}{F(n)} 100
$$

Moreover, percent weights of limestone and quartz in recycle are:

$$
L_{R}(n)=\frac{R_{L}(n)}{R(n)} 100
$$

And

$$
Q_{R}(n)=\frac{R_{Q}(n)}{R(n)} 100
$$

The undersize screen product, $P$, can be obtained from a simple mass balance: 


$$
P(n)=R(n-1)+F(n)-R(n)=M-R(n)
$$

In which the amount of limestone is:

$$
P_{L}(n)=R_{L}(n-1)+F_{L}(n)-R_{L}(n)
$$

And the amount of quartzite is:

$$
P_{Q}(n)=R_{Q}(n-1)+F_{Q}(n)-R_{Q}(n)
$$

Hence, the percentage limestone and quartzite in the screened product are:

$$
\begin{aligned}
& L_{P}(n)=\frac{P_{L}(n)}{P(n)} 100 \\
& Q_{P}(n)=\frac{P_{Q}(n)}{P(n)} 100
\end{aligned}
$$

The general algorithm for cycle-wise simulation of locked-cycle tests with binary mixture feeds is given in eqns. (9) to (38). One computes the set of system variables in these equations until the values converge as the steady-state is reached. However, in order to implement the simulation, it is first necessary to account for interaction phenomena in an explicit fashion. Recalling eqn. (2), the function in eqn. (27) becomes

$$
\phi_{L}\left[t \sum_{k=j}^{n} S_{L}(k)\right]=\exp \left[G_{L} t \sum_{k=j}^{n} S_{L}(k)+H_{L} \frac{t^{2}}{t}\left[\sum_{k=j}^{n} S_{L}(k)\right]^{2}\right]
$$

The absolute values of $G$ s are invariably much larger than $\mathrm{Hs}$, which in fact tend to zero. Therefore, eqn. (39) may be simplified somewhat to:

$$
\phi_{L}\left[t \sum_{k=j}^{n} S_{L}(k)\right]=\exp \left[G_{L} t \sum_{k=j}^{n} S_{L}(k)+H_{L} \frac{t^{2}}{t}(n-j+1)^{2}\right]
$$

Next, the following empirical expression is proposed to relate the energy split factor SL with the composition of the mill charge:

$$
\frac{S_{L}^{*}-S_{L}(k)}{S_{L}^{*}-1}=W_{L} M_{L}^{a}(k)+\left(1-W_{L}\right) M_{L}^{b}(k)
$$

where $S_{L}^{*}$ is greater than one and is the maximum possible split factor as the amount of the softer component becomes vanishingly small, $W_{L}$ lies between zero and one, and $a$ and $b$ are exponents. Similarly, for quartzite in eqn. (28):

$$
\phi_{Q}\left[t \sum_{k=j}^{n} S_{Q}(k)\right]=\exp \left[G_{Q} t \sum_{k=j}^{n} S_{Q}(k)+H_{Q} \frac{t^{2}}{t}(n-j+1)^{2}\right]
$$

and the energy split factor $S_{Q}(k)$ is:

$$
\frac{s_{Q}(k)-s_{Q}^{*}}{1-S_{Q}^{*}}=W_{Q} M_{Q}^{c}(k)+\left(1-W_{Q}\right) M_{Q}^{d}(k)
$$


where $S_{Q}^{*}$ is again the minimum split factor as the quartzite amount in the mixture becomes vanishingly small, $W_{Q}$ lies between zero and one, and $c$ and $d$ are exponents.

\section{Parameter Estimation and Model Validation}

As discussed in detail in our previous papers, grinding experiments to test the model were carried out dry in a laboratory stainless steel mill $25.4 \mathrm{~cm}$ in diameter and $27.9 \mathrm{~cm}$ in length using 2.54-cm diameter stainless steel balls as grinding media [10]. Standard mill operating conditions included a mill speed of $54 \mathrm{rpm}$ ( $60 \%$ of mill critical speed), $30 \mathrm{~kg}$ of stainless steel balls (roughly $50 \%$ of the struck volume of the mill) and $100 \%$ filling of void volume of the struck ball charge by the feed material. The torque drawn by the mill was measured and recorded using a BHL torquesensing system. The size distribution of the mill contents was determined by a standard wet-dry sieving technique. Determination of the size distribution of each component involved assaying each size fraction by chemical methods or by determination of densities.

Locked-cycle tests were carried out using a 2 min grind cycle with single-component feeds of limestone and quartzite, as well as with their mixtures in weight ratios of 1:3, 1:1 and 3:1. In one set of experiments 1:1 limestone-quartzite feed was used for the first twelve cycles followed by a switch to 1:3 feed. The results are reported in the form of total recycle as a function of the cycle number. After a 2 min grind, the product was sieved on a 65-mesh (210 ${ }^{\wedge} \mathrm{m}$ ) screen for $10 \mathrm{~min}$ and the oversize material returned to the mill, together with the required amount of fresh feed to maintain the total solids volume constant in each cycle.

The lumped grinding parameters $G$ and $H$ were estimated separately from the lockedcycle test results using single-component feed of limestone or quartzite. The root mean square error between experimental values of the recycle and those computed from the algorithm in eqns. (1) to (8) was minimized by optimizing the parameters $G$ and $H$ by a direct search technique [15].

Figure 1 shows that the simulated recycles are in very good agreement with the experimental results for both pure limestone and quartzite feeds.

Using these estimates of $\mathrm{Gs}$ and $H s$, another search was conducted to find the best values of IPs, $S^{*}$ s and the exponents in the expressions for the energy split factors in eqns. (41) and (43). Here the error function was based on mixture feed data and the locked-cycle grinding algorithm in eqns. (9) to (43).

Figure 2 shows a comparison between the experimental and the simulated values of the recycles for two feeds, namely, 1:1 and 3:1 limestone-quartzite mixtures. The agreements are quite satisfactory. A more stringent test of the simulation scheme is demonstrated in Fig. 3, where the feed composition is altered drastically in the thirteenth grinding cycle from a ratio of 1:1 to $1: 3$. Clearly, the simulation is able to track the transient well; indeed, the overall agreement is quite remarkable. 


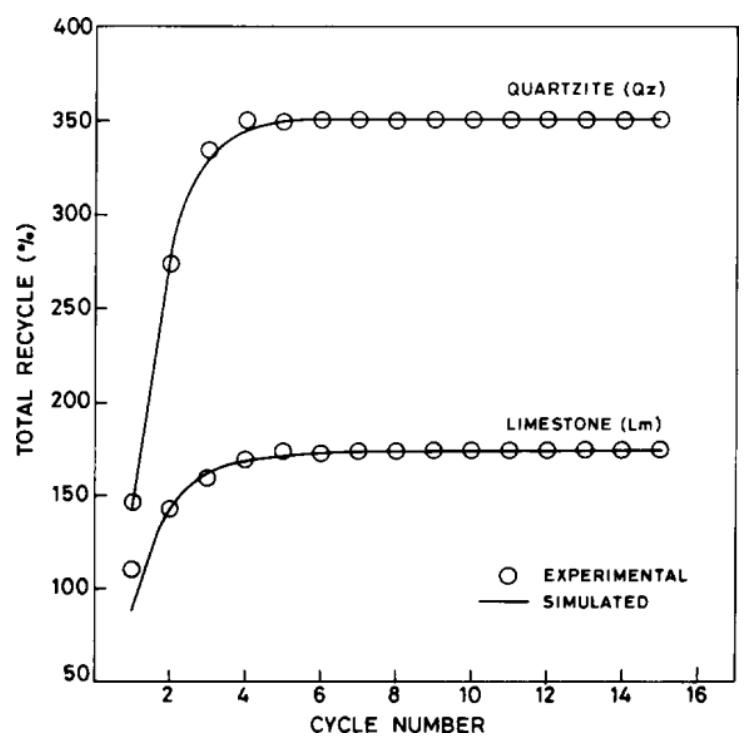

Fig. 1. Experimental and simulated recycles for the locked-cycle grinding of pure limestone and quartz feeds.

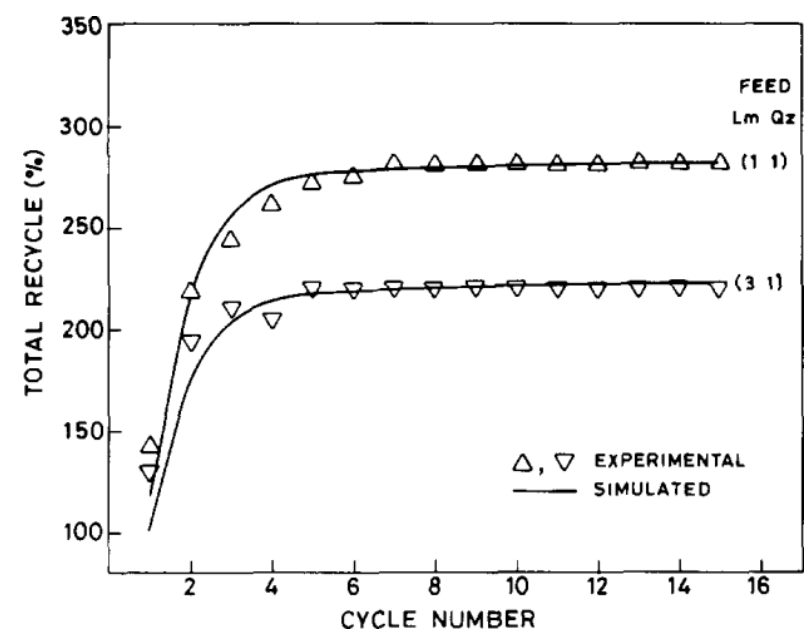

Fig. 2. Experimental and simulated recycles for $1: 1$ and 3:1 limestone-quartzite mixtures.

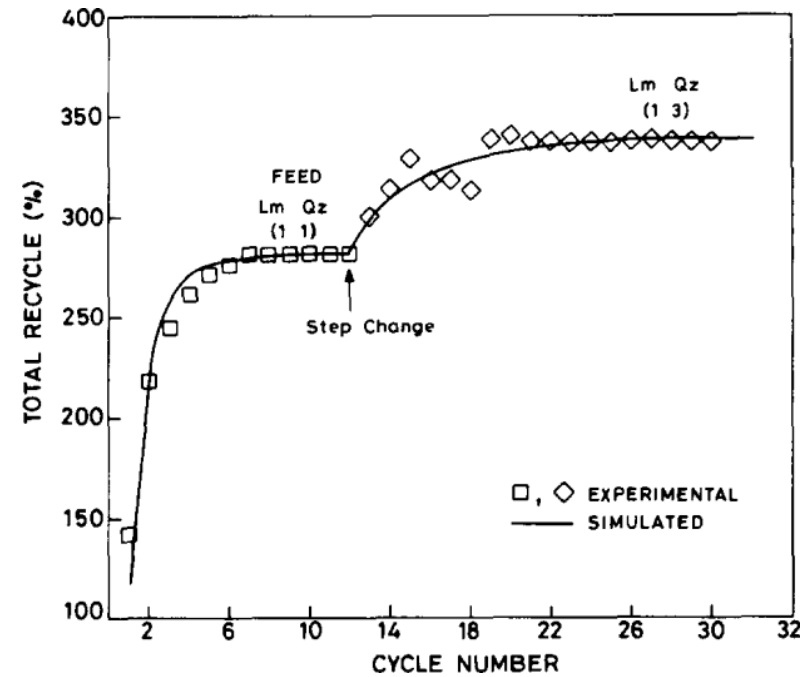

Fig. 3. Experimental and simulated recycles for the locked-cycle grinding of 1:1 limestonequartzite mixtures with a stepchange to a feed ratio of 1:3. 
It is therefore reasonable to conclude that the simulation algorithm presented here is reliable and could permit a detailed examination of the likely behavior of the grinding circuit in the face of changing/fluctuating feed characteristics and operating conditions.

\section{Simulation Results}

Figure 4 presents the simulated locked-cycle tests for different feeds ranging from pure limestone to pure quartzite, with seven mixture compositions in between. Note that the higher the proportion of the hard mineral in the feed, the steeper the change in the circulating load from cycle to cycle in the transient regime of the test. Figure 5 shows the steady-state percent recycle ratio as a function of feed composition. For the most part, the recycle ratio varies almost linearly with composition except when the feed contains more than $80 \%$ quartzite.

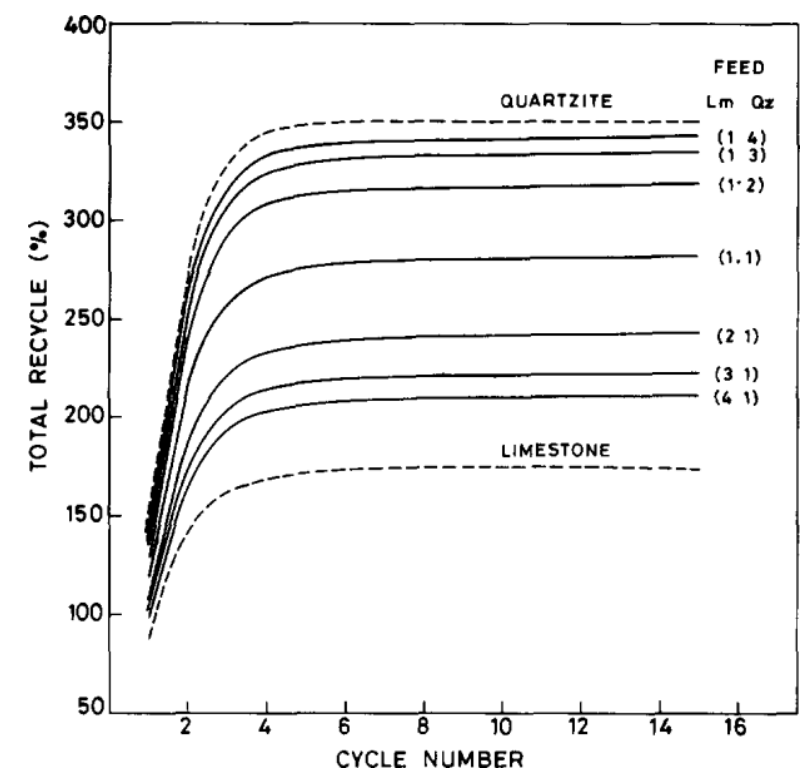

Fig. 4. Simulated total recycle for the locked-cycle grinding of a variety of feeds ranging from pure quartzite to pure limestone

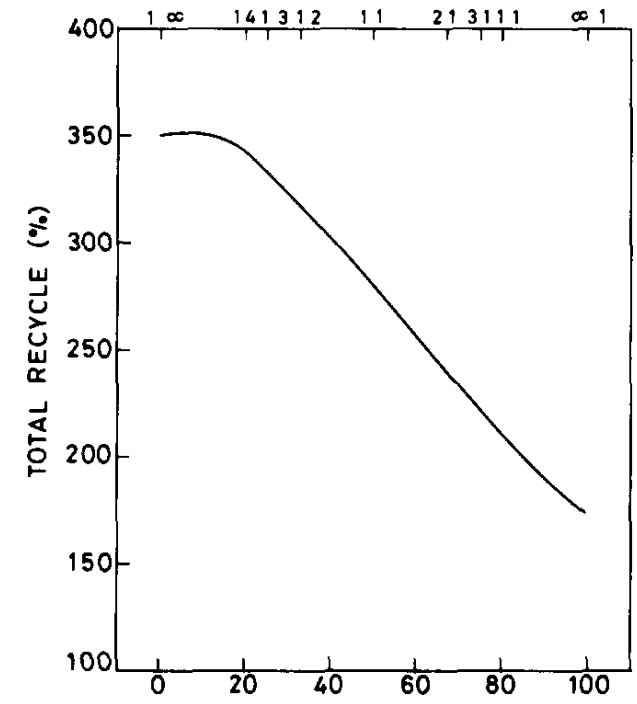

Fig. 5. The simulated steady-state recycle ratio as a function of fee competition. 
A major source of circuit instability is the fact that the hardness and grindability of a natural ore feed undergo more or less continuous random changes. This characteristic can be mimicked to some extent by varying at random the composition of the feed mixture of hard and soft minerals between a specified band width. Figure 6 illustrates the fluctuations in recycle loads when random variations in the range of \pm 0.1 units are imposed on the composition of three feeds, namely, 1:3, 1:1 and 3:1 limestone-quartz mixtures. For example, in the case of 1:1 feed the quartz content at the start of each grinding cycle is permitted to vary between 40 and $60 \%$ by the expedient of adding a uniformly distributed random component to the feed composition. A number of conclusions can be derived from this figure. Feed comprised predominantly of the hard component exhibits the most fluctuations. In fact, there seems to be a slight long-term upward drift in the recycle amount. The correlation between the two cyclewise series pertaining to composition and recycle is quite complex, and shows an erratic time lag which is conceivably further accentuated in an actual grinding circuit, where the residence time distribution is not exactly of a plug- flow type. Moreover, additional time is needed for material transport through the recycle loop.

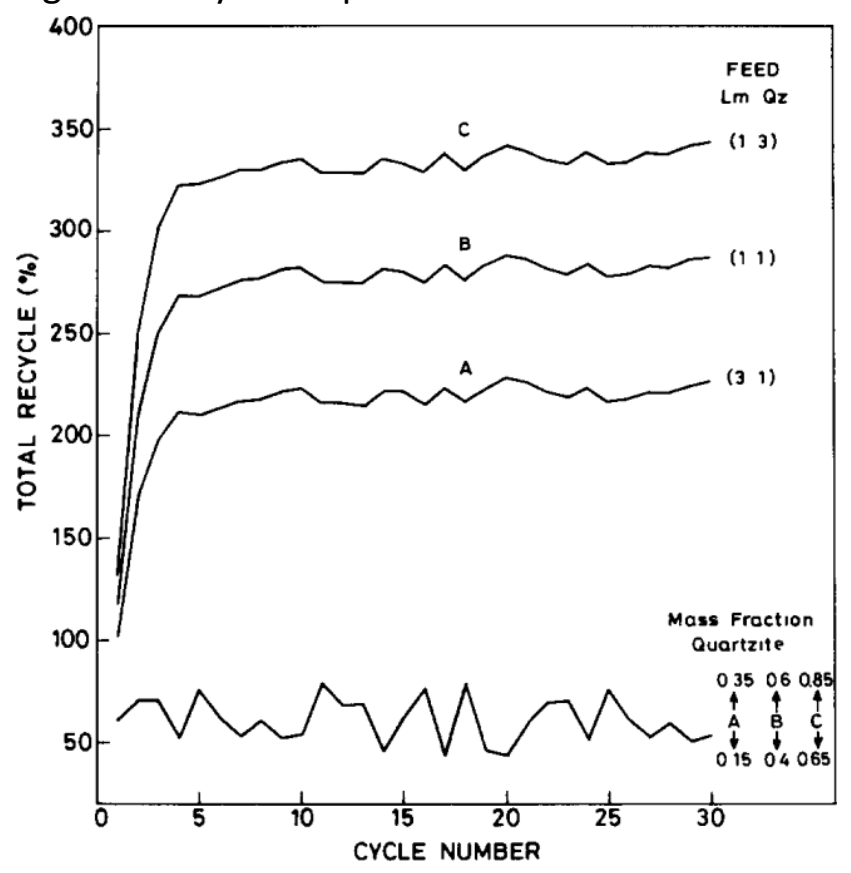

Fig. 6. Fluctuations in the recycle loads with random fluctuations in the feed composition.

Because of the built-in nature of the crusher circuit, it simply cannot provide a mill feed of invariant size consist. Therefore, another source of circuit instability is the varying fractions of feed components that are coarser than the mesh-of-grind, $\mathrm{rL}$ and $\mathrm{rQ}$. In the next set of simulations, the fraction of feed coarser than the mesh-of-grind in the two components was allowed to vary at random in a band of \pm 0.1 units. Because now $r s$ are not fixed, it is necessary to shift them within the summation sign as $r L(/)$ and $r Q(j)$ in eqns. (27) and (28). As shown in Fig. 7, these two random series apparently have a reinforcing effect and the recycle tends to fluctuate rather strongly, leading to a highly unstable circuit even in the case of 3:1 limestone- 
quartzite feed which ordinarily possesses inherently superior dampening characteristics, as is evident in Fig. 6.

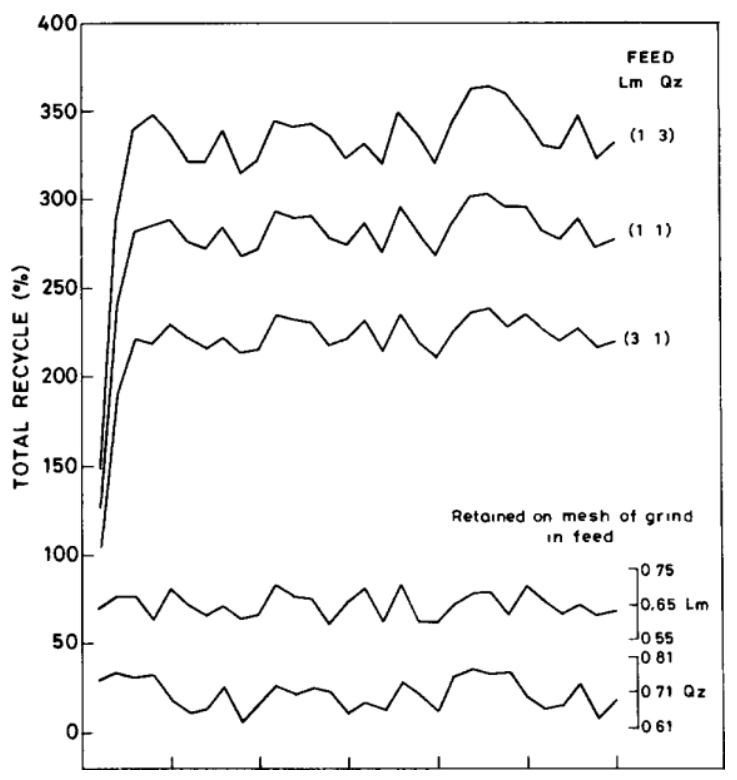

Fig. 7. Fluctuations in the recycle loads with random fluctuations in the mesh of grind.

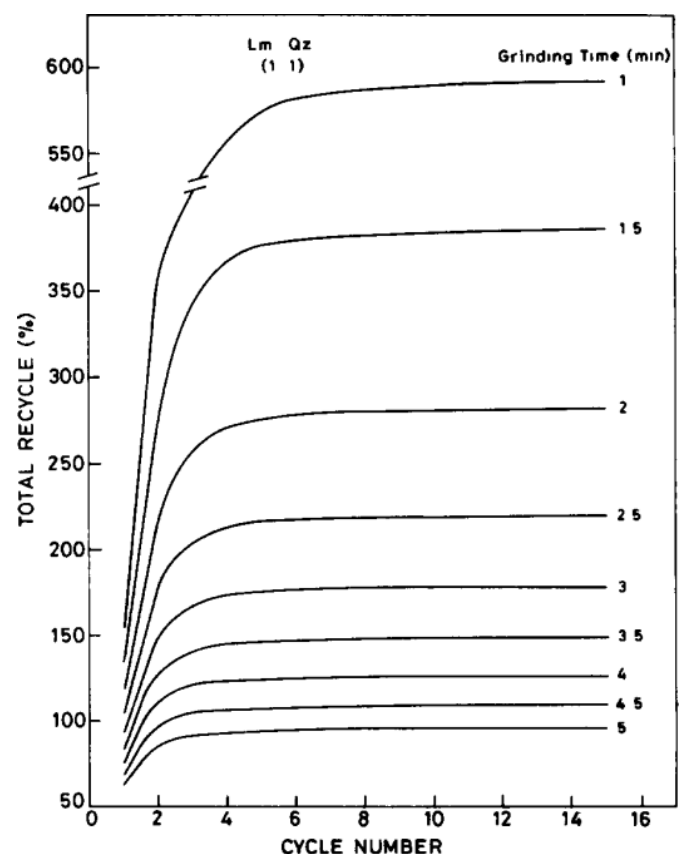

Fig. 8. The recycle load as a function of cycle number for various grinding times.

Figure 8 shows the simulated locked-cycle grinding tests using 1:1 limestone-quartzite feed when the time of the grinding cycle was varied from 1 to $5 \mathrm{~min}$ in increments of $0.5 \mathrm{~min}$. Figure 9 shows how the steady- state recycle changes as a function of the grinding time. Note that the recycle initially drops steeply as cycle time is increased, but tends to level off at prolonged grinding times. This indicates that it would be much more difficult to control a circuit which operates under a fast throughput rate than one with a long residence time. 
As feed rate changes, due to surging phenomena in the crusher circuit, limitations in the accuracy of the mill feeder and variations in the recycling amount, the residence time in the mill also changes in an erratic manner. Here again, time $t$ is inserted within the summation sign as $t(k)$ in eqns. (27) and (28). Figure 10 shows the effect of +0.1 min random variations imposed on

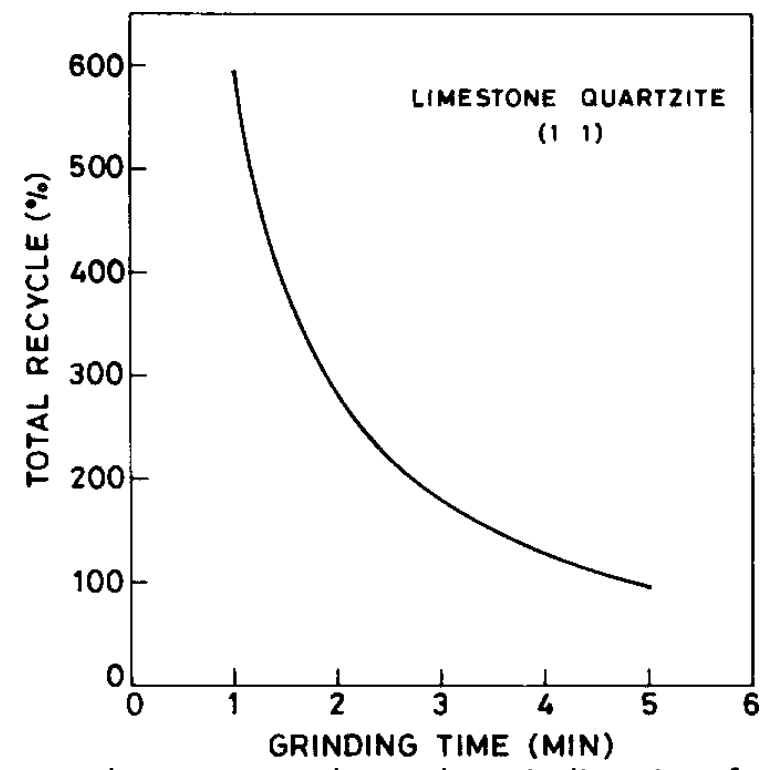

Fig. 9. Dependence of the steady-state recycle on the grinding time for the grinding of 1:1 limestone-quartzite feeds.

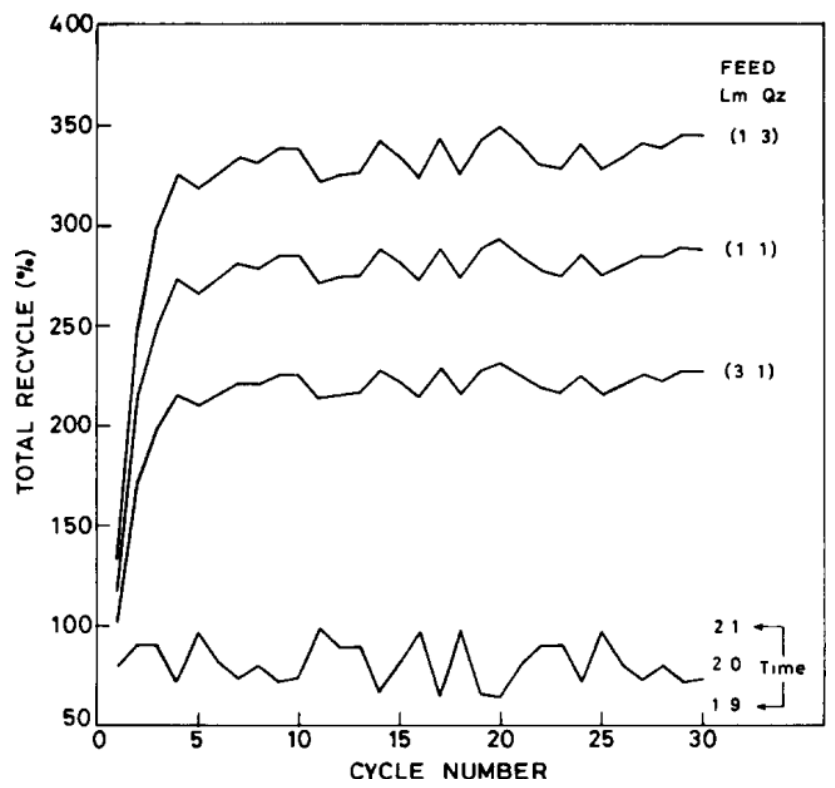

Fig. 10. Effect of random variations imposed on the 2 min nominal grinding cycle time on the stability of the total recycle for different feeds.

the 2 min nominal grinding time for each cycle. The fluctuations are least in the case of 3:1 limestone-quartzite feed, and are distinctly larger and comparable for 1:1 and 1:3 feeds. Figure 11 illustrates the effect of band width over which the grinding time is permitted to vary for 1:1 
limestone-quartzite feed. It is clear that even with a $+10 \%$ range, which is not unrealistically large for an industrial circuit, the stability is degraded considerably.

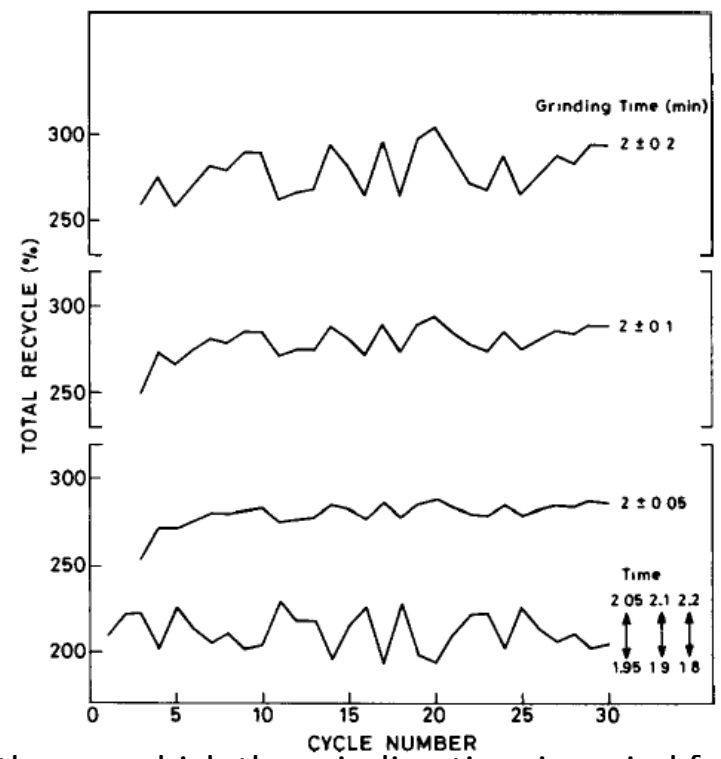

Fig. 11. Effect of band width over which the grinding time is varied for the grinding of $1: 1$ limestone-quartzite feeds.

Finally, Figure 12 shows a long-term change in feed composition from 1:1 limestonequartzite mixture to 1:3 or 3:1 mixture for anywhere from one to five cycles before reverting

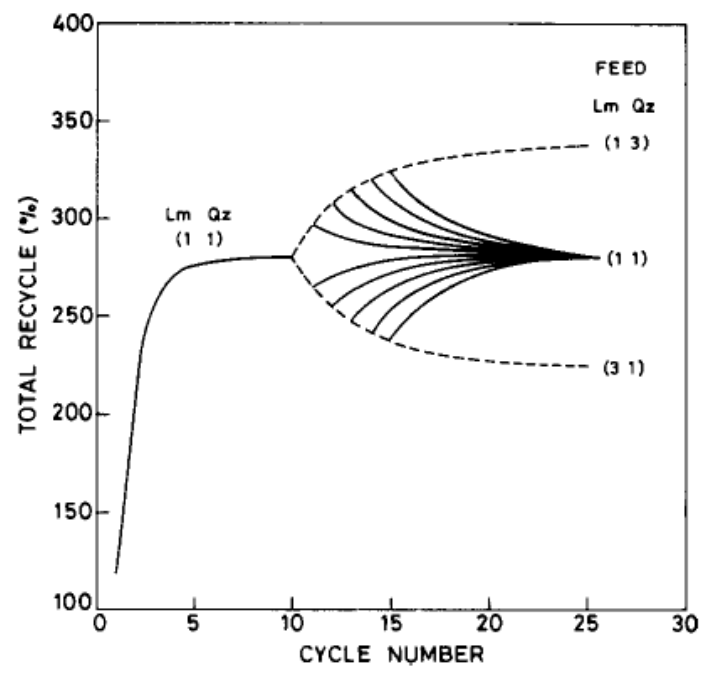

Fig. 12. The effect of a long-term change in feed composition, followed by the return back to a 1:1 feed mixture.

back to 1:1 mixture feed. It is now possible to follow the transient behavior of the recycle. Somewhat surprisingly, the duration of the disturbance does not seem to have any pronounced effect on the time required for the system to settle back to steady state again. 


\section{Conclusions}

A grinding circuit is subject to many kinds of random and cyclic disturbances, which reside in the changing feed characteristics, variable residence time, erratic classifier performance, pile-up and surging of material in the circuit loop, among other reasons. The most direct consequence of these disturbances is on the recycling amount, which invariably fluctuates to a lesser or greater degree with or without long-term cyclic drift. When acting in conjunction with each other, these disturbances moreover should, if anything, accentuate the problem further. This is the primary reason why it is difficult to maintain the stability of a grinding circuit and why its control is such a complex task indeed.

We have presented a relatively simple scheme that allows us to examine the behavior of an industrial closed-loop grinding circuit with plug flow transport through the mill. Recent simulation studies by us based on detailed mathematical models of grinding kinetics with arbitrary residence time distributions in the mill exhibit remarkably similar results. Although for demonstration purposes the results presented here are based on imposition of only one kind of disturbance at a time, the simulator is however much more general in scope. For example, it is possible to impose simultaneously any combination of disturbances. Moreover, the effect of an imperfect classifier - not included in the present study - can be readily incorporated into the simulation scheme. In addition, with only minor modifications we can also simulate the alternative situation in which the new feed rather than the total mill feed is kept constant, or even when the new feed rate is variable, for effecting an appropriate control strategy. Finally, the scheme is simple enough to be implemented on an on-line small computer for real time monitoring and control of the circuit.

\section{Acknowledgement}

The authors express appreciation to the United States Bureau of Mines for support of this research under the Generic Mineral Technology Center Program in Comminution (Grant No. G1175149).

\section{References}

1 W. L. Maxson, F. Cadena and F. C. Bond, Trans. AIME, 112 (1934) 130.

2 E. Onuma, H. Asai and G. Jimbo, /. Chem Eng Jpn., 9 (1976) 485.

3 R. T. Hukki and H. Allenius, Trans. AIME, 241 (1968) 482.

4 C. A. Rowland and D. M. Kjos, in A. L. Mular and R. B. Bhappu (eds.), Mineral Processing Plant Design, 2nd edn., AIME, New York, 1980, p. 239

5 G. D. Gumtz and D. W. Fuerstenau, Trans AIME, 247 (1970) 330.

6 P. C. Kapur, Trans. Inst. Min. Metall, 79 (1970) C103. 
7 P. C. Kapur and M. Rahman, Trans. Inst. Min Metall., 80 (1971) C-74.

8 V. K. Karra, CIM Bull, 74 (1981) 195.

9 K. S. Venkataraman and D. W. Fuerstenau, Powder Technol, 39 (1984) 133.

10 D. W. Fuerstenau and K. S. Venkataraman, Int. J. Mm Process., 22 (1988) 105.

11 P. C. Kapur and D. W. Fuerstenau, Powder Technol., 59 (1989) 39.

12 P. C. Kapur and D. W. Fuerstenau, Int. J. Min Process, 24 (1988) 125.

13 P. C. Kapur, Min. Met Process. J, 5 (1988) 133

14 H. E. Rose and S. Matsumura, Trans. Inst. Min Metall., 94 (1985) C40.

15 R Luus and T. H. Jaakola, Al CHE J., 19 (1973) 760. 